\title{
EL ROL DE LAS UNIVERSIDADES \\ EN EL DESARROLLO SOCIOCULTURAL \\ DE LAS REGIONES DEL PAÍS*
}

Roberto Escobar

Enrique Sáez

\section{MEDIR LA CULTURA}

La cultura de una sociedad proviene de un conjunto abstracto de valores que se manifiestan siempre en acciones sociales concretas; por ello es posible hacer comparaciones de la forma con la cual una institución y las personas que la forman, generan acciones para conservar, aumentar, difundir y analizar los "objetos" y los "productos" de una cultura determinada, en relación a igual acción de otras instituciones o sociedades.

La conservación y difusión cultural —en las universidades-se realiza a través de la vida académica y se refleja:

a) en la docencia, por la eficacia de la transmisión cultural recíproca profesoralumno,

b) por el tipo y amplitud de sus bibliotecas y colecciones y la intensidad de su uso,

c) por la actividad artística que proyecta valores y símbolos del subconsciente colectivo,

d) por las publicaciones que fijan el pensamiento y la creación,

e) por los diferentes tipos de relación entre la universidad y personas de la Región y el país,

f) a través de radio, televisión y otros medios con los cuales la universidad se dirige a toda la población intra y extramuros.

* Informe final del Proyecto de Investigación $\mathrm{N}^{\circ}: \mathrm{H}-2106-8512$ realizado entre abril 1985 y agosto 1987, en la Universidad de Chile. 
El objeto de esta investigación es comparar la actividad cultural de las universidades en relación a su Región. La comparación permite visualizar la posiblidad de que las universidades actuales puedan cumplir satisfactoriamente su cometido.

No es posible establecer niveles de "bueno" o de "malo" puesto que la acción cultural es ilimitada y siempre positiva aun si su nivel es bajo, pero se consideran como factores adversos al desarrollo social de una Región que su universidad no aporte alementos culturales comparables a lo que parece normal dentro de las tradiciones y tendencias del país.

Los parámetros que se consideraron son:

- población regional: total, estudiantil, urbana y rural;

- público potencial para la acción cultural regional;

- dotación de profesores de jornada completa y parcial;

- bibliotecas y colecciones;

- museos, salas para espectáculos, galerías de arte, etc.;

- estaciones de radio y televisión;

- publicaciones.

Las fuentes bibliográficas utilizadas se enumeran en el anexo bibliográfico y provienen de las mismas universidades estudiadas, Consejo de Rectores, autores especializados, Instituto Nacional de Estadísticas y otros.

Los investigadores sostuvieron diversas entrevistas con rectores, profesores y especialistas en asuntos universitarios, quienes aportaron importantes enfoques para la realización del trabajo. Se aprovecha esta oportunidad de manifestar a todos los que colaboraron el reconocimiento y la gratitud de los autores.

\section{CRONOLOGÍA DE LA VIDA UNIVERSITARIA}

A partir de la fundación de la Universidad de Chile en 1842 se puede agrupar la actividad universitaria nacional en los períodos siguientes:

I. $1842-1900$.

Etapa de expansión técnica y cientifica.

La U. de Chile funciona como coordinadora de todo el proceso educacional chileno y como su centro de investigación científica y tecnológica, por ello su influencia es decisiva en el desarrollo del país.

En este período las artes se enseñan y practican en academias es:atales autónomas: Academia de Bellas Artes (fundada en 1847), Escuela de Arquitectura (1849), Conservatorio Nacional de Música (1851), todos los cuales pasarian eventualmente a formar parte de la Universidad de Chile. Además se establece la Escuela de Artes y Oficios (1857) que pasará a formar parte de la Universidad Técnica del Estado más adelante. 
Coincidiendo con la expansión de las universidades pontificias en el mundo, se produce en Chile una escisión política entre grupos que se identifican con la Iglesia y los laicos que favoreció la creación de la Pontificia Universidad Católica de Chile en 1888, cuya acción cultural, en esta primera etapa, es muy discreta comparada a la amplia acción ejercida por el Estado a través de la Universidad de Chile y las Academias de Arte.

II. 1901-1930.

Etapa de adaptación de la vida chilena a la evolución social de postguerra.

En este período se producen: expansión demográfica y mayor amplitud en la actividad regional.

Se crean, por iniciativa privada, la Universidad de Concepción en 1922 y la Universidad Católica de Valparaíso en 1924, absorbiendo una Escuela de Arquitectura independiente que existía en forma esporádica, en Valparaíso.

Termina este período con la promulgación del Estatuto Orgánico de la Enseñanza Superior en Chile, mediante el Decreto Ley $\mathrm{N}^{\circ} 4807$, que es el primer paso a la independencia entre las universidades existentes, si bien la $U$. de Chile aún ejercía un control general sobre la educación superior.

Al fin del período la presión social sobre la Universidad se hace sentir con muchos trastornos que llevan a reformular las políticas generales y a establecer un nuevo Estatuto para la U. de Chile, que la regirá hasta 1972.

\section{1931-1950}

Etapa de inicio de la Extensión Universitaria.

El aislamiento académico con Europa que se agudiza durante la II Guerra Mundial, impulsa el desarrollo de la actividad cultural universitaria y el inicio de los contactos académicos con los EE.UU.

El desarrollo industrial chileno también repercute en las universidades inaugurando una especial preocupación por la tecnología, lo que se vio consolidado con la creación de la Universidad Técnica Federico Santa Maria y la proyección de la Escuela de Artes y Oficios hacia la carrera de Ingeniero Industrial, primer paso a la consolidación definitiva de las Escuelas Industriales de las Provincias y las Escuelas de Minas para formar, con carácter nacional, la Universidad Técnica del Estado en 1947.

En esta forma se delimitaba, en cierta forma, el campo cultural entre las dos universidades estatales nacionales. La U. de Chile actuaba en Santiago y la UTE en provincias, la $U$. de Chile dirigía, prácticamente la actividad humanista y artística y la UTE la tecnológica industrial. 
En 1934, se normaliza la actividad administrativa y académica en la $U$. de Chile y se crea el Servicio de Extensión Universitaria, concepto nuevo en Chile pero ya practicado desde el XIX en Inglaterra y otros países.

Era la manifestación de un deseo del gobierno de fortalecer la vida social y cultural de las provincias y llevarles el beneficio de la difusión de los valores intelectuales y artísticos de la U. de Chile.

Nacieron así las Escuelas de Temporada y los Cursos de Extensión, el "Tren de la Cultura" que viajaba con vagones especiales habilitados como salas de clase, se empieza a utilizar la radio con fines universitarios y se fortalece el esfuerzo editorial de la U. de Chile.

Ante este esfuerzo nacional, la U. Católica crea también su organismo de Extensión y fortalece su trabajo editorial. La seriedad del trabajo de Extensión se puede medir con la personalidad y prestigio de quienes dirigían la Extensión: Amanda Labarca y luego Francisco Galdames en la U. de Chile, y Jaime Eyzaguirre en la U. Católica.

\section{1951-1960.}

Ampliación regional del sistema académico.

El término de la ll Guerra Mundial marca una época de eclosión cultural en Chile. La cantidad y calidad de las obras intelectuales y artísticas realizadas en Chile en la década del 50 es marcadamente superior a todo lo que se conoció antes y después de ese decenio.

Concordante con lo anterior la U. de Chile crea Colegios Regionales, primero y Sedes Universitarias, después, en 8 Regiones fuera de Santiago, con cursos regulares y actividades culturales propias.

Se organiza el Consejo de Rectores, primer esfuerzo para coordinar, distribuiry estimular la investigación científica por la asignación de fondos especiales, y se diversifica la actividad académica separando, en cierto modo, la docencia de la investigación y de la extensión.

La regionalización universitaria se ve fortalecida con el esfuerzo privado y una corporación funda la Universidad Austral en 1956. La U. Católica abre cursos y establece sedes en Concepción, Los Ángeles y Villarrica, y la UCV inicia cursos en Antofagasta en 1959 los que formarán la Universidad del Norte en 1963, dirigidas por los jesuitas.

La UTE inaugura una Sede en Santiago cuya moderna edificación viene a ser símbolo del espíritu de los tiempos. 
Periodo de politización de las universidades.

Durante este período se acentúa la idea de que el control político de las universidades es una forma de controlar políticamente el país. Esto tomó diversas formas: creación de organismos autónomos o semiautónomos, dependientes de las universidades, llamados "institutos" o "centros" destinados a difundir posiciones políticas, publicaciones y formación de monitores ideológicos; contratación de personas como "profesores" para encubrir su verdadera tarea que no era académica; utilizar las Escuelas de Arte como centros de propaganda mediante el "arte comprometido"; la formación de grupos paramilitares entre los alumnos y la participación activa de los estudiantes en movimientos laborales.

En 1968 y años siguientes se introduce la "Reforma Universitaria" de intención política, con el propósito de impedir el control de las universidades, ya fuera por el Gobierno, las Fundaciones o la Iglesia. Ya en 1972 la actividad universitaria prácticamente se detiene y se diluye en prolongadas asambleas, huelgas y tomas de edificios.

La vida cultural de las universidades se interrumpe y se dispersa.

En 1973 la distribución de sedes universitarias, a lo largo del país era la siguiente:

\begin{tabular}{lccccccccc}
\hline Región & U. CH. & UTE & U. CON & UTFSM & AUST & UC & UCV & UN & Total \\
\hline I & 2 & 1 & - & - & - & - & - & - & 3 \\
II & 1 & 1 & - & - & - & - & - & 1 & 3 \\
III & - & 1 & - & - & - & - & - & - & 1 \\
IV & 1 & 1 & - & - & - & - & - & - & 2 \\
V & 1 & - & - & 2 & - & - & 1 & - & 4 \\
RM & 4 & 1 & - & - & - & 1 & - & - & 6 \\
VI & - & - & - & - & - & - & - & - & - \\
VII & 1 & 1 & - & - & - & 1 & - & - & 3 \\
VIII & 1 & 1 & 2 & 1 & - & 2 & - & - & 7 \\
IX & 1 & 1 & - & - & - & 2 & - & - & 4 \\
$X$ & 1 & 1 & - & - & 1 & - & - & - & 3 \\
XI & - & - & - & - & - & - & - & - & - \\
XII & - & 1 & - & - & - & - & - & - & 1 \\
\hline Total & 13 & 10 & 2 & 3 & 1 & 6 & 1 & 1 & 37 \\
\hline
\end{tabular}


VI. 1974-1980.

Reestructuración del sistema universitario.

Restablecido el orden en el pais, se inicia un proceso de recuperación de la vida universitaria; ello significó importantes cambios en la estructura orgánica, revisión de estatutos y políticas, las universidades son regidas por Rectores-Delegados, quienes reemplazan a los organismos colegiados.

Durante este período se desea intensificar la vida cultural y se recuperan las actividades de Extensión, alcanzando un nivel más activo que el que había tenido en un largo tiempo. Particularmente se presta mucha atención a las actividades regionales, las que empiezan a tener un buen nivel de apoyo desde Santiago.

Durante este período se estudian activamente las posibles medidas de regionalización universitaria.

VII. 1981 en adelante.

Etapa de regionalización universitaria.

A partir de 1981 se ponen en práctica las nuevas disposiciones legales que establecen las llamadas "carreras universitarias", la obligatoriedad de dar grados académicos, y las sedes regionales de la U. de Chile y la UTE pasan a formar Universidades estatales regionales. Se separan las carreras pedagógicas para crear dos universidades especiales. Se agrupan las carreras "técnicas" en Institutos Profesionales.

Junto a lo anterior se admite la creación de universidades privadas sin subvención fiscal pero con el control de una universidad examinadora.

Se multiplican los Institutos Profesionales y Técnicos de origen municipal y privado, permitiéndose incluso la formación de profesores de Media y Básica en institutos particulares pero vedándolo a las universidades de Chile y de Santiago.

El desarrollo de la Televisión a lo largo del país y el mejoramiento técnico de los canales universitarios ha constituido un cambio importante en la forma de vinculación entre las universidades y su público extramuros, situación a la cual aún no se le ha dado suficiente importancia.

El sistema universitario, en los años 1985-87, período en el cual se realizó este estudio, se ve debilitado y falto de políticas claras en su tarea formadora de personas y en la difusión de valores sociales y culturales.

Esta indefinición de políticas y metas se refleja en una distribución presupuestaria inadecuada y en las frecuentes e irracionales demostraciones de protesta universitaria.

El cuadro de establecimientos universitarios en 1987 es el siguiente, consignando su origen: 


Región Universidad Origen

\begin{tabular}{|c|c|c|c|}
\hline I & $\begin{array}{l}\text { Tarapacá } \\
\text { Arturo Prat }\end{array}$ & $\begin{array}{l}\text { (2) } \\
\text { (2) }\end{array}$ & \\
\hline 11 & $\begin{array}{l}\text { Antofagasta } \\
\text { Norte }\end{array}$ & $\begin{array}{l}\text { (2) } \\
\text { (3) }\end{array}$ & \\
\hline III & Atacama & (2) & \\
\hline IV & $\begin{array}{l}\text { La Serena } \\
\text { Norte (Sede) }\end{array}$ & $\begin{array}{l}\text { (2) } \\
\text { (3) }\end{array}$ & \\
\hline V & $\begin{array}{l}\text { Valparaíso } \\
\text { Playa Ancha } \\
\text { UCV } \\
\text { UTFSM } \\
\text { Escuela de Negocios }\end{array}$ & $\begin{array}{l}\text { (2) } \\
(2) \\
(3) \\
\text { (4) } \\
\text { (5) }\end{array}$ & \\
\hline RM & $\begin{array}{l}\text { Chile } \\
\text { USACH } \\
\text { Metropolitana } \\
\text { Católica } \\
\text { Central } \\
\text { Diego Portales } \\
\text { Gabriela Mistral } \\
\text { Tarapacá (Sede) } \\
\text { Escuela de Negocios (Sede) }\end{array}$ & $\begin{array}{l}\text { (1) } \\
(2) \\
(2) \\
(3) \\
(5) \\
(5) \\
(5) \\
(2) \\
(5)\end{array}$ & \\
\hline VI & No hay Universidad & & \\
\hline VII & $\begin{array}{l}\text { Talca } \\
\text { Católica (Sede) }\end{array}$ & $\begin{array}{l}\text { (2) } \\
\text { (3) }\end{array}$ & \\
\hline VIII & $\begin{array}{l}\text { Biobío } \\
\text { Concepción } \\
\text { Concepción (Sede), Chillán } \\
\text { UTFSM (Sede) } \\
\text { Católica (Sede) }\end{array}$ & $\begin{array}{l}(2) \\
(4) \\
(4) \\
\text { (4) } \\
\text { (3) }\end{array}$ & \\
\hline IX & $\begin{array}{l}\text { Frontera } \\
\text { Católica (Sede) }\end{array}$ & $\begin{array}{l}\text { (2) } \\
\text { (3) }\end{array}$ & \\
\hline$x$ & Austral & (4) & $\begin{array}{l}\text { NOTA: } \\
(1)=\text { Universidad Nacional }\end{array}$ \\
\hline $\mathrm{XI}$ & No hay Universidad & & $\begin{array}{l}(2)=\text { Universidad Estatal Regional } \\
(3)=\text { Universidad Católica con subvención estata }\end{array}$ \\
\hline XII & Magallanes & (2) & $\begin{array}{l}(4)=\text { Universidad privada con subvención estatala } \\
(5)=\text { Universidad privada sin subvención estatal }\end{array}$ \\
\hline
\end{tabular}


La evolución del sistema universitario entre 1973 y 1987 es el siguiente:

19731987

TOTAL

Universidades

Sedes

ESTATALES

Universidades

Sedes

214

$23 \quad 15$

$\begin{array}{rr}8 & 24 \\ 37 & 32\end{array}$

PRIVADAS

SUBVENCIONADAS

Universidades

Sedes

PRIVADAS

NO SUBVENCIONADAS

Universidades

5 Sedes
19731987

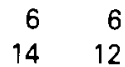

12 
Tabla 1

CARACTERÍSTICAS DE LA ACTIVIDAD CULTURAL

$\begin{array}{ll}\text { Investigación cultural } & =\text { INV. CULT. } \\ \text { Intensidad } & =\text { INT. } \\ \text { Calidad } & =\text { CAL. } \\ \text { Conservación cultural } & =\text { CONS. CULT. } \\ \text { Difusión } & =\text { DIF. CULT. } \\ \text { Actividad creativa } & =\text { ACT. CREA. }\end{array}$

\begin{tabular}{|c|c|c|c|c|c|c|}
\hline Región & Universidad & $\begin{array}{l}\text { INV. } \\
\text { INT. }\end{array}$ & $\begin{array}{l}\text { CULT. } \\
\text { CAL. }\end{array}$ & $\begin{array}{l}\text { CONS. } \\
\text { CULT. }\end{array}$ & $\begin{array}{l}\text { DIF. } \\
\text { CULT. }\end{array}$ & $\begin{array}{l}\text { ACT. } \\
\text { CREA. }\end{array}$ \\
\hline 1 & $\begin{array}{l}\text { Tarapacá } \\
\text { Arturo Prat }\end{array}$ & $\begin{array}{l}\mathrm{B} \\
\mathrm{C}\end{array}$ & $\begin{array}{l}\mathrm{B} \\
\mathrm{B}\end{array}$ & $\begin{array}{l}\mathrm{B} \\
\mathrm{C}\end{array}$ & $\begin{array}{l}\mathrm{B} \\
\mathrm{C}\end{array}$ & $\begin{array}{l}\mathrm{B} \\
\mathrm{C}\end{array}$ \\
\hline 11 & $\begin{array}{l}\text { Norte } \\
\text { Antofagasta }\end{array}$ & $\begin{array}{l}\text { C } \\
\text { A }\end{array}$ & $\begin{array}{l}\text { A } \\
\text { B }\end{array}$ & $\begin{array}{l}\mathrm{B} \\
\mathrm{C}\end{array}$ & $\begin{array}{l}\mathrm{B} \\
\mathrm{C}\end{array}$ & $\begin{array}{l}\mathrm{B} \\
\mathrm{B}\end{array}$ \\
\hline III & Atacama & C & A & B & $\mathrm{C}$ & $\mathrm{C}$ \\
\hline IV & La Serena & B & A & B & B & A \\
\hline v & $\begin{array}{l}\text { Valparaíso } \\
\text { UCV } \\
\text { UTFSM } \\
\text { Playa Ancha }\end{array}$ & $\begin{array}{l}A \\
B \\
B \\
C\end{array}$ & $\begin{array}{l}\mathrm{C} \\
\mathrm{C} \\
\mathrm{B} \\
\mathrm{C}\end{array}$ & $\begin{array}{l}\mathrm{C} \\
\mathrm{C} \\
\mathrm{C} \\
\mathrm{C}\end{array}$ & $\begin{array}{l}C \\
A \\
B \\
C\end{array}$ & $\begin{array}{l}\mathrm{C} \\
\mathrm{B} \\
\mathrm{C} \\
\mathrm{B}\end{array}$ \\
\hline RM & $\begin{array}{l}\text { Chile } \\
\text { Católica } \\
\text { USACH } \\
\text { Metropolitana }\end{array}$ & $\begin{array}{l}A \\
A \\
C \\
C\end{array}$ & $\begin{array}{l}A \\
C \\
C \\
B\end{array}$ & $\begin{array}{l}A \\
C \\
B \\
C\end{array}$ & $\begin{array}{l}A \\
A \\
C \\
C\end{array}$ & $\begin{array}{l}A \\
A \\
C \\
A\end{array}$ \\
\hline VI & (no hay) & & & & & \\
\hline VII & Talca & $\mathrm{C}$ & B & $\mathrm{C}$ & C & $\mathrm{B}$ \\
\hline VIII & $\begin{array}{l}\text { Concepción } \\
\text { Biobío }\end{array}$ & $\begin{array}{l}A \\
C\end{array}$ & $\begin{array}{l}\mathrm{A} \\
\mathrm{C}\end{array}$ & $\begin{array}{l}A \\
C\end{array}$ & $\begin{array}{l}A \\
C\end{array}$ & $\begin{array}{l}A \\
C\end{array}$ \\
\hline IX & Frontera & C & C & c & $\mathrm{C}$ & $\mathrm{C}$ \\
\hline$x$ & Austral & A & A & $B$ & B & $B$ \\
\hline$X I$ & (no hay) & & & & & \\
\hline XII & Magallanes & $\mathrm{B}$ & $\mathrm{C}$ & $\mathrm{B}$ & $\mathrm{C}$ & c \\
\hline
\end{tabular}

$\bar{A}=$ actividad amplia y satisfactoria; $B=$ dentro del promedio del país; $C=$ insuficiente para la región. 
Tabia II

INVESTIGACIÓN DEL CONOCIMIENTO

Y LA CULTURA: INTENSIDAD

\begin{tabular}{llc}
\hline Grado & Universidad & Índice \\
\hline A: & Valparaíso & 10 \\
& Chile & 10 \\
& Austral & 10 \\
& Antofagata & 9 \\
Católica & 9 \\
& Concepción & 9 \\
& & \\
B: $\quad$ Tarapacá & 8 \\
& La Serena & 8 \\
& UCV & 8 \\
UTFSM & 8 \\
& Magallanes & 8 \\
& Norte & 7 \\
& Arturo Prat & \\
C: & Playa Ancha & 6 \\
& Talca & 6 \\
& Frontera & 6 \\
Atacama & 6 \\
Santiago & 5 \\
Biobio & 5 \\
Metropolitana & 3 \\
& &
\end{tabular}

La dedicación a la investigación del conocimiento y la cultura se ha medido como la proporción entre el número de profesores ( $\mathrm{J} / \mathrm{C}$ equivalentes) dividido por el número de alumnos, multiplicado por 100. 
Tabla III

INVESTIGACIÓN DEL CONOCIMIENTO

Y LA CULTURA: CALIDAD

\begin{tabular}{llr}
\hline Grado & Universidad & Índice \\
\hline A: & Norte & 22,3 \\
& Austral & 4,5 \\
& Concepción & 3,7 \\
& Atacama & 3,5 \\
& La Serena & 3,4 \\
& Chile & 3,0 \\
& & \\
B: & Metropolitana & 2,7 \\
& Tarapacá & 2,6 \\
& Antofagasta & 2,2 \\
& Arturo Prat & 1,8 \\
& UTFSM & 1,7 \\
& Talca & 1,4 \\
& & \\
C: & UCV & 1,1 \\
& Frontera & 1,1 \\
& Católica & 1,0 \\
& Playa Ancha & 1,0 \\
Biobío & 0,6 \\
& Magallanes & 0,6 \\
Santiago & 0,6 \\
& Valparaíso & 0,5
\end{tabular}

Expresa la dedicación académica por la proporción entre profesores de jornada completa y parcial, expresada en $\mathrm{J} / \mathrm{C}$ equivalentes. 
Tabla IV

CONSERVACIÓN CULTURAL

\begin{tabular}{ll}
\hline Grado & Universidad \\
\hline A: & $\begin{array}{l}\text { Chile } \\
\text { Concepción }\end{array}$ \\
& \\
B: & Tarapacá \\
& Norte \\
& Atacama \\
& La Serena \\
& Santiago \\
& Austral \\
& Magallanes \\
& \\
& Arturo Prat \\
& Antofagasta \\
& Valparaíso \\
& UCV \\
& UTFSM \\
& Playa Ancha \\
& Católica \\
& Metropolitana \\
& Talca \\
& Biobío \\
& Frontera \\
&
\end{tabular}

El interés y el esfuerzo en la conservación del patrimonio cultural se mide por los museos, científicos y artísticos, colecciones especializadas, salas destinadas a exponer trabajos intelectuales y artísticos, teatros, salas de concierto y otros.

Se considera en Grado A a las universidades que cuentan con una infraestructura completa y colecciones de interés especial. En Grado B las que cuentan con uno o dos museos especializados pero que no disponen de salas $u$ otros medios para difundir valores culturales. En Grado $\mathrm{C}$ se han clasificado las universidades no equipadas para tareas relevantes de conservación cultural, sólo colecciones menores y una sala de espectáculos. 
Tabla V

DIFUSIÓN CULTURAL

\begin{tabular}{ll}
\hline Grado & Universidad \\
\hline A: & Chile \\
& Católica \\
& UCV \\
& Concepción \\
& \\
& Tarapacá \\
B: & Norte \\
& La Serena \\
& Austral \\
& UTFSM \\
& \\
& Arturo Prat \\
& Antofagasta \\
& Atacama \\
& Valparaíso \\
& Playa Ancha \\
& Metropolitana \\
& Talca \\
& Biobío \\
& Frontera \\
& Magallanes \\
& USACH \\
& \\
&
\end{tabular}

Mide la actividad realizada a través de medios de radio, televisión, editorial de libros y publicaciones periódicas, junto a otras formas de extender masivamente el interés y el conocimiento cultural y científico.

Se han clasificado en Grado $A$ a aquellas universidades que usan estos medios en forma sistemática e intensa. En Grado $B$ a aquellas que operan sólo algunos de estos medios y en menos intensidad. En Grado $\mathrm{C}$ aquellas cuyo trabajo de difusión es limitado y esporádico. 
Tabla VI

ACTIVIDAD CREATIVA

\begin{tabular}{ll}
\hline Grado & Universidad \\
\hline A: & Chile \\
& Concepción \\
& La Serena \\
& Católica \\
& Metropolitana \\
& \\
& Tarapacá \\
B: & UCV \\
& Playa Ancha \\
& Talca \\
& Norte \\
& Antofagasta \\
& Austral \\
& \\
& Arturo Prat \\
& Valparaíso \\
& UTFSM \\
& USACH \\
& Biobío \\
& Frontera \\
& Atacama \\
& Magallanes
\end{tabular}

Mide la instalación y promoción de conjuntos destinados a la creación, interpretación y aprendizaje de las artes.

Se clasificó como Grado $A$ a aquellas universidades que mantienen conjuntos musicales estables, grupos de danza y teatro, escuelas de música y de plástica. Como Grado B, con actividades estables pero menos completas, solo una escuela de arte, plástica o música. En Grado $\mathrm{C}$ se consideraron las que tienen actividades artísticas esporádicas y sin escuelas estables de artes. 
Tabla VII

POBLACIÓN UNIVERSITARIA REGIONAL Y URBANA EN 1984

\begin{tabular}{|c|c|c|c|}
\hline Región & $\begin{array}{l}\text { Población } \\
\text { Universitaria }\end{array}$ & $\begin{array}{c}\text { Población } \\
\text { Regional }\end{array}$ & $\begin{array}{c}\text { Población } \\
\text { Urbana }\end{array}$ \\
\hline
\end{tabular}

\begin{tabular}{lccccc}
\hline & $(1)$ & $(2)$ & $(3)$ & $(4)$ & $(5)$ \\
I & 3,8 & 1,28 & 1,40 & 297,2 & 270,7 \\
II & 9,1 & 2,53 & 3,23 & 358,9 & 281,9 \\
III & 0,9 & 0,47 & 0,74 & 188,2 & 112,2 \\
IV & 4,0 & 0,92 & 2,00 & 433,9 & 199,1 \\
V & 15,3 & 1,21 & 1,80 & $1.266,9$ & 852,3 \\
RM & 45,0 & 0,98 & 1,04 & $4.612,8$ & $4.337,7$ \\
VI & - & - & - & 604,1 & \\
VII & 5,2 & 0,33 & 0,70 & 747,9 & 157,0 \\
VIII & 16,8 & 1,08 & 1,96 & $1.558,9$ & 856,7 \\
IX & 6,3 & 0,66 & 3,80 & 705,8 & 163,7 \\
X & 3,7 & 0,43 & 1,28 & 856,5 & 289,6 \\
XI & - & - & - & 69,5 & \\
XII & 0,9 & 0,66 & 1,06 & 134,6 & 84,5 \\
\hline Nacional & 110,9 & 0,99 & 1,46 & $11.161,1$ & $7.614,4$
\end{tabular}

(1) $=$ población estudiantil, en miles.

(2) $=\%$ de alumnos universitarios respecto de la población regional.

(3) $=\%$ de alumnos universitarios respecto de la población urbana de la región.

(4) $=$ población regional, en miles.

(5) = población urbana, en miles.

Fuentes: INE Y Consejo de Rectores.

\section{CONCLUSIONES}

La razón objetiva para fundar las universidades chilenas ha sido planteada con espíritu positivista, para ayudar al desarrollo científico y tecnológico, llegando a confundir el papel de las universidades con la formación profesional exclusivamente; sin embargo, el desempeño de ellas demuestra que su verdadera orientación y finalidad ha sido mejorar los niveles sociales y culturales del país y las provincias.

El presente análisis, al establecer el grado de concordancia entre lo que podríamos llamar: Potencialidad, Efectividad y Creatividad en la tarea de investigar, 
conservar, difundir y enseñar los valores del conocimiento y la cultura arrojan el siguiente resultado*:

1. Universidades que demuestran un alto grado de concordancia y que, en consecuencia, aportan satisfactoriamente al desarrollo regional y nacional:

Chile

Concepción

Austral

La Serena

Tarapacá

2. Universidades con actividades culturales no integradas, énfasis parcial en algunos aspectos, pero inadecuado para las necesidades:

Católica

Norte

Antofagasta

Metropolitana

Atacama

Talca

3. Universidades que descansan en la actividad cultural de otras de la misma Región o de otra (particularmente notorio en la $V$ Región que descansa en la labor de la RM):

Valparaiso

UTFSM

UCV

Playa Ancha

Arturo Prat

USACH

Biobio

Frontera

Magallanes

Analizando algunos factores que influyen en la efectividad cultural de las universidades surgen: los aspectos de condición urbano-rural, el tamaño del alumnado y el financiamiento.

Hay universidades cuyo emplazamiento urbano les comunica características propias de un entorno cultural "directo", es decir, con espectáculos de arte, difu-

* Potencialidad se calculó por la suma de índices 1 y 2 de la Tabla 1; Efectividad por la su ma de 3 y 4 ; y Creatividad por la suma de 2 y 5 . 
sión científica y humanista a través de la Extensión y el aporte cultural de publicaciones y periódicos, etc.

Las características rurales requieren otro tipo de acción, las universidades deben disponer de hogares estudiantiles para los de fuera, programas de radio y TV dirigidos a un público rural, enseñanza a distancia, etc. En Chile no hay universidades propiamente organizadas para actividades en el medio rural. Esto contribuye a la alta migración hacia los centros poblados y a la mayor velocidad de evolución del sector urbano respecto del rural.

Midiendo la condición de ruralidad por la proporción entre la población regional total y la urbana se obtiene:

A: Público universitario predominantemente urbano:

Región I $=1,10$

Región II $=1,27$

Región Met. $=1,06$

B: Público universitario equilibrado entre lo urbano y lo rural:

Región III $=1,68$

Región IV $=2,18$

Región $\mathrm{V}=1,76$

Región VIII $=1,80$

Región XII $=1,59$

C: Público universitario predominantemente rural:

Región VII $=4,76$

Región IX $=4,31$

Región $X \quad=2,96$

Al comparar estas condiciones con los grupos señalados arriba como deficientes, se observa que las universidades predominantemente rurales son deficientes culturalmente y que su política y metodología debe reformularse a las exigencias rurales; éstas son, particularmente: Talca, Frontera y Austral y en menor grado: Magallanes, Biobio, Valparaiso y Playa Ancha.

Respecto al tamaño del alumnado, se observa que a medida que aumenta el alumnado hay una tendencia a mejorar el nivel cultural de la universidad.

Parece que no es posible que una universidad con menos de 3.000 alumnos pueda alcanzar un nivel normal, este es el caso de las siguientes universidades:

Arturo Prat

Atacama

Playa Ancha

Magallanes 
Las universidades con más de 10.000 alumnos presentan condiciones culturales más favorables, especialmente:

Chile

Católica

Concepción

La excepción a este grupo es USACH que descansa en la labor de la $U$. de Chile en la Región Metropolitana.

No hay universidades en Chile con alumnado entre 10.000 y 7.500 , y entre 7.500 y 5.000 se sitúan dos universidades cuyo desempeño cultural es mediano:

Norte

UCV

Entre 3.000 y 5.000 alumnos, se sitúan la mayoría de las universidades, algunas de las cuales, en realidad sirven a otras Regiones que aquellas donde están ubicadas. Esto se hace más claro al comparar la población estudiantil con la regional.

El promedio nacional es que $0,99 \%$ de la población es estudiantil. La condición en cada Región es la siguiente:

$\begin{array}{ll}\text { Región II } & 2,53 \% \\ \text { Región I } & 1,28 \% \\ \text { Región V } & 1,21 \%\end{array}$

Las universidades de estas tres Regiones reciben más alumnos que los del lugar, producto de una migración estudiantil.

$\begin{array}{ll}\text { Región VIII } & 1,08 \% \\ \text { Región Met. } & 0,98 \% \\ \text { Región IV } & 0,92 \%\end{array}$

Las universidades de estas otras tres Regiones están dentro de promedio nacional de $0,99 \%$.

$\begin{array}{ll}\text { Región IX } & 0,66 \% \\ \text { Región XII } & 0,66 \% \\ \text { Región III } & 0,47 \% \\ \text { Región X } & 0,43 \% \\ \text { Región VII } & 0,33 \%\end{array}$

Estas cinco Regiones no alcanzan a dar un servicio universitario acecuado a su población.

Finalmente, en las Regiones VI y XI no hay actividad universita- a. Esto es sorprendente respecto de la VI, ya que tiene más de 600.000 habitantes. o a ue daría 
alrededor de 6.000 alumnos universitarios potenciales, más que suficiente para una universidad de buen tamaño.

Frente a lo anterior resulta inexplicable que la Región III con menos de 200.000 habitantes y la XII con menos de 150.000 tengan una universidad cada una.

Por razones geopolíticas es evidente que se necesita una universidad en Magallanes a cualquier costo, pero la justificación de ella requiere aumentar sensiblemente su tamaño.

El caso de Valparaíso señala que sus universidades están sobredimensionadas para la demanda regional y dan escaso servicio al público rural de la $V$ Región. En general las universidades de la $V$ Región son alternativas para estudiantes de otras regiones, lo que se confirma por el bajo aporte cultural que esas universidades dan a su Región.

Con referencia al financiamiento cabe señalar que nunca habrá suficiente financiamiento para las posibilidades educacionales de un país. La tarea de distribuir recursos entre los diferentes niveles de educación da para muchas polémicas, especialmente la asignación de fondos a universidades estatales y subvencionadas.

Los autores de esta investigación desconocen - como ya se ha dicho- los detalles de los fondos de que dispone cada universidad, pero es evidente que el Estado ha disminuido su aporte y que la regionalización universitaria, a partir de 1981 ha significado un deterioro de la actividad cultural en las universidades, especialmente en las que no son de un tamaño suficiente.

Contrario a lo que habitualmente se sostiene, los autores creen que el desarrollo social y cultural homogéneo del país es una preocupación casi exclusiva de las universidades, las que deben realizar un trabajo de investigación, conservación, difusión y enseñanza culturales, como parte del desarrollo integral.

La investigación universitaria es sólo posible a las universidades más grandes, no porque tengan más dinero, sino porque tienen más personal académico y porque generan más recursos propios.

La distribución centralizada de fondos sólo resuelve una parte del problema, ya que es evidente que las universidades más alejadas de los centros poblados deben tener, proporcionalmente, más académicos de alto nivel que las más urbanas.

La comparación que ofrece este trabajo hace resaltar el hecho de que las universidades que han dado cabida a la enseñanza artística y a su difusión, por caro que resulte, demuestran una clara superioridad en su aporte al conocimiento y a la cultura. Es el caso de las cinco universidades que tienen un alto grado de concordancia en su actividad cultural:

Chile

Concepción

Austral 


\section{La Serena}

\section{Tarapacá}

Uno de los efectos detrimentales de la regionalización de las universidades estatales ha sido la de hacer más difícil el cumplimiento de sus tareas como centros de desarrollo regional. Lo anterior unido a la disminución de sus posibilidades de generar financiamiento propio, por no contar con el personal idóneo ni la infraestructura necesaria.

Si bien las universidades más grandes pueden generar más recursos que las medianas, aun en términos proporcionales, las pequeñas simplemente no pueden generar recursos.

\section{BIBLIOGRAFÍA}

La universidad y la producción de certificados educacionales, José Joaquín Brunner, Documento de Trabajo $N^{\circ} 166$, FLACSO, 43 pp.

Argumento y realidad en la universidad chilena, José Joaquín Brunner, Documento de Trabajo $N^{\circ} 160$, Flacso, Santiago, Chile, 1982.

Informe sobre e/ desarrollo y e/ estado actual del sistema universitario en Chile, José Joaquín Brunner, Documento de Trabajo $\mathrm{N}^{\circ}$ 227, FlACso, Santiago, Chile, mayo de 1985.

Cuadernos de la Universidad de Chile, números 1-3, 1981-1983.

Guia de Ingreso a las Instituciones de Educación Superior Privada, 1983-1984.

Guía de Ingreso a los Institutos Profesionales Privados, 1984.

Informaciones Pedagógicas Universitarias, Universidad de Chile, Dirección General Académica, números 1-18, años 1974-1981.

Informativo Estadístico, Admisión y Selección de Alumnos, Universidad de Chile, números 1-2; años 1982-1983.

Informativo Estadístico, Servicio de Títulos y Grados, Universidad de Chile, años 19781983.

Monografías, Servicio de Registro y Selección de
Alumnos, Universidad de Chile, números 1-18, años 1977-1983.

Revista de la Universidad de Chile, Vicerrectoría de Extensión, Universidad de Chile, años 1971-1984.

Boletín del Ministerio de Educación, números 10-29, años 1980-1982.

Boletín Estadístico de Matrícula, Universidad de Chile, números 2-4, años 1982-:983

Boletín Informativo del Consejo de Rectores, números 1-48, años 1971-1980

Boletín Informativo del Servicio ce Jesarrollo Docente, Universidad de Chi.e, - i meros 1 13 , años 1977-1980.

Boletín Informativo del Servicio de Se ección y Registro de Estudiantes. Univers dad de Chile, números 1-9, años $1976 \cdot 984$

Universidad y Desarrollo Regiona: Formación de recursos humanos e inves: gación para e/ desarrollo regional, Progra $a=\equiv \_-C$ VDA, Santiago de Chile, marzo ce $\cdot 98 ., 487$ pp.

Ciencia, tecnología y arte en la un versidad de Chile. Panorama actua! L-versidad de Chile, Editorial Universitar a. Santiago de Chile, 1982, 92 pp.

Identidad Nacional, VII Jornadas Jea onales de 
Cultura, Facultad de Filosofía, Humanidades y Educación, Universidad de Chile, Editorial Universitaria, Santiago, Chile, 1982, $190 \mathrm{pp}$.

La Universidad Contemporánea. Un intento de análisis empírico, Schiefelbein, Ernesto y Mc Ginn, Noel, Corporación de Promoción Universitaria, Santiago, Chile, 1974, 313pp.

Desarrollo Científico-Tecnológico y Universidad, Boeninger, Edgardo, Corporación de Promoción Universitaria, Santiago, Chile, 1973, 388 pp.

Diccionario bibliográfico de miembros de la Universidad de Chile, Ávila Martel, Alamiro de, en Anales de la Universidad de Chile, quinta serie, número 3 , Santiago, Chile, noviembre de 1983, 381 y ss.

La educación permanente: un derecho de todos, en Crónica de la Unesco, número 11, vol. xvIII, París, noviembre de 1972, pp. 217 y ss.

Educación permanente y educación sobre población, Paul Regand, unesco, 1976, 28 pp.

Actividades Académicas 1979, Prorrectoría de la Universidad de Chile, Santiago, Chile, julio de 1980.

Anuario de Carreras y Programas Académicos 1980, Universidad de Chile, Santiago, Chile, 279 pp.

La formulación de objetivos y politicas de la Universidad de Chile, Hernán García Vidal, enero de 1979, 15 pp.

Reseña histórica de la Universidad de Chile, Alamiro de Ávila Martel, Santiago, Chile, 1979, 78 pp.

Publicaciones originadas en 101 proyectos de investigación y finalizados en 1982, Dirección General Académica, Universidad de Chile, número 5, 1983, 204 pp.

Publicaciones Científicas de la Universidad de Chile 1981, Departamento de Desarrollo de la Investigación, Santiago, Chile, 1983, 257 pp.

Universidad de La Serena, Guía de Ingreso 1985.

Universidad de Concepción, Guía Académica 1985.

Universidad de Concepción, Escuela de Graduados, Catálogo General 1984-1985.

Historia de los 90 años de la Pontificia Universi- dad Católica de Chile, Ricardo Krebs y otros, Revista Universitaria, número 1 , Santiago, Chile, 1978.

La Administración de la Investigación Científica en la Pontificia Universidad Católica de Chile, Raúl Cañas, Revista Universitaria, número 4, Santiago, Chile, octubre de 1980 , pp. 47-59.

Investigación y Postgrado en una Universidad Católica, Ricardo Couyoumdjian y Rafael Vicuña, Revista Universitaria, número 6 , Santiago, Chile, octubre de 1981, pp. 131142.

El ingreso a la Universidad de Chile y a las carreras de Pedagogia en el año 1978, Victor Nazar, Corporación de Promoción Universitaria, Colección Estudios Sociales, número 25 , tercer trimestre 1980 , Santiago, Chile, pp. 135-172.

Evolución histórica de los intentos de descentralización educacional en Chile, Hernán Vera, Corporación de Promoción Universitaria, Colección Estudios Sociales, número 42, 1984, Santiago, Chile, pp. 161-164.

La Universidad Latinoamericana. Visión de una década, Patricio Dooner e Iván Lavados, Corporación de Promoción Universitaria, Santiago, Chile, 666 pp.

El rol de la ciencia en el desarrollo, Ataliva Amengual y Jaime Lavados (editores), Corporación de Promoción Universitaria, Santiago, Chile, 1978, 271 pp.

Una visión de la comunidad cientifica nacional, Corporación de Promoción Universitaria y Academia de Ciencias del Instituto de Chile, Santiago, Chile, 1982.

La educación superior en Chile, Corporación de Promoción Universitaria, enero de 1984, 172 pp.

La Universidad Latinoamericana en la década de/ 80, Luis Scherz (editor), Corporación de Promoción Universitaria, 1976, 289 pp.

Guía de Post-Grado en las Universidades Chilenas 1984, Consejo de Rectores, Santiago, Chile.

Nueva institucionalidad universitaria, Asesoria Jurídica, Consejo de Rectores, Santiago, Chile, 1981, 50 pp. 
Nueva legislación universitaria chilena, Secretaría General, Consejo de Rectores, Santiago, Chile, 1981, 129 pp.

Catálogo de jornadas, seminarios y otras actividades universitarias 1980, Secretaría General, Consejo de Rectores, Santiago, Chile, 1980, 91 pp.

Publicaciones universitaria chilenas 1980, Secretaría General, Consejo de Rectores, Santiago, Chile, 1980, 43 pp.

Profesiones de Post-Grado, Secretaría General, Consejo de Rectores, Santiago, Chile, 1983, 204 pp.

Anuario Estadístico 1980, Consejo de Rectores, Santiago, Chile, 1980, 285 pp.

Anuario Estadístico 1981, Consejo de Rectores, Santiago, Chile, 1981, 213 pp. y anexos.

Anexo Anuario Estadístico 1981, Consejo de Rectores, Santiago, Chile, 1981, 74 pp.

Anuario Estadístico 1982, Consejo de Rectores, Santiago, Chile, 1982.

Anuario Estadístico 1984, Consejo de Rectores, Santiago, Chile, 1984.

Nómina de Institutos Profesionales y Centros de Formación Técnica aprobados por el Ministerio de Educación Pública, Documento $\mathrm{N}^{\circ}$ 17, Consejo de Rectores, Santiago, Chile, marzo-agosto de 1982.

Antecedentes nacionales e internacionales sobre grados académicos y estudios de PostGrado, tomo I, Universidad de Santiago de Chile, mayo de 1981, 198 pp.

Análisis y evaluación de los antecedentes, tomo II, Universidad de Santiago de Chile, junio de 1981, 23 pp.

Cuenta de Rectoría, Pontificia Universidad Católica de Chile, tomo I, Santiago, Chile, 1981.

Cuenta de Rectoría, Pontificia Universidad Católica de Chile, tomo ॥, Santiago, Chile, 1982.

Actividades de investigación 1979-1980, Pontificia Universidad Católica de Chile, Santiago, Chile, 1980.

Catálogo de Asignaturas, Universidad Austral de Chile, 1980, 268 pp.

Catálogo de Asignaturas, Universidad Austral de Chile, 1981, $273 \mathrm{pp}$.

Catálogo de Asignaturas, Universidad Austral de Chile, 1982, 244 pp.
Catálogo de Post-Grado, 1983, Universidad Austral de Chile, 155 pp. Memoria 1984, Universidad Austral de Chile, 2 vols., 173 pp.

Programa de Graduados, Universidad de Concepción, Chile, 1980.

Programa de Graduados, Universidad de Concepción, Chile, 1979.

Escuela de Graduados, Universidad de Concepción, Chile, categoría general, 1984-1985.

Cooperación Técnica Internacional y Universitaria, Carlos Martínez Sotomayor, Universidad de Concepción, documento $\mathrm{N}^{\circ} 68$, 1982.

Memoria Anual, Universidad de Talca, 1982, 142 pp.

Andes 1981-1982, Universidad de La Frontera, documento $\mathrm{N}^{\circ} 2,216 \mathrm{pp}$.

Universidad de La Frontera, 1982, 24 pp.

Andes 1983, Universidad de La Frontera, Chile, 1983.

Boletín Bibliográfico 1982, Universidad de La Frontera, Chile.

Programación Docente, Planes y Programas de Estudio 1982, Universidad Técnica Federico Santa María, Valparaíso, 1982, 143 pp.

Memoria de Tesis de Graduación, Trabajos de Título 1978-1981, Universidad Técnica Federico Santa María, Valparaíso, agosto de 1982, 73 pp.

Proyectos de Investigación 1981, Universidad Católica de Valparaíso, Chile, 1981

Bases para la estrategia del desarrollo de la Universidad del Norte, Rectoría, Universidad del Norte, Chile, 1980, 45 pp

Actividades de Investigación, Universidad de Tarapacá, Arica, 1982, 111 pp.

Actividades de Investigación, Universidad de Tarapacá, Arica, Chile, 1983.

Leyes, Decretos y Reglamentos, Universidad de Chile, 2 volúmenes, Santiago, Chile, 1953.

Estadistica de los Titulos y Grados otorgados por la Universidad de Chile, Santiago, Chile, 1981, $365 \mathrm{pp}$.

Catálogo General 1985-1986, Pontificia Universidad Católica de Chile, Consejo de Rectores, pp. 51-67, 241-305.

Estatuto de la Universidad de Chile, Universidad de Chile, enero de 1982. 
Matrícula de los alumnos de pregrado de la Universidad de Chile año 1981, Universidad de Chile, Servicio de Selección y Registro de Estudiantes, Boletín Estadístico de Matrícula, julio de 1982.

Procedencia Geográfica y Escolar de los Alumnos Ingresados a Primer Nivel, Universidad de Chile 1980, Universidad de Chile, Vicerrectoría de Asuntos Académicos, Servicio de Selección y Registro de Estudiantes, noviembre de 1980.

Estudios de Post-Grado y post título ofrecidos en la Universidad de Chile, Vicerrectoría de Asuntos Académicos, Servicio de DesarroIlo Docente, marzo de 1980.

Bases para una politica y planificación de la ciencia y la tecnologia en Chile, Universidad de Chile, Facultad de Ciencias Físicas y Matemáticas, Centro de Planeamiento, $200 \mathrm{pp}$.

La Universidad de Chile en el contexto universitario nacional, Prorrectoría, Servicio de Planificación, diciembre de 1979, 130 pp.

Anuario de Carreras y Programas Académicos 1980, Prorrectoría, Servicio de Planificación, 1980, $279 \mathrm{pp}$.

Matricula de los Alumnos de Pregrado de la Universidad de Chile año 1979, Vicerrectoría de Asuntos Académicos, Servicio de Selección y Registro de Estudiantes, mayo de 1980.

Proceso de Admisión a la Educación Superior.
Postulantes y postulaciones año académico 1982, Universidad de Chile, Dirección de Servicios Estudiantiles, Servicio de Selección y Registro de Estudiantes, octubre de 1982.

Proceso de Admisión a la Educación Superior. Postulantes y postulaciones año académico 1983, Universidad de Chile, Dirección General Académica, Servicio de Selección y Registro de Estudiantes, agosto de 1983.

Publicaciones Cientificas de la Universidad de Chile, 1981, Universidad de Chile, Dirección General Académica, Departamento de Desarrollo de la Investigación, octubre de 1983,257 pp.

Proyectos de Investigación 1982, Universidad de Chile, Departamento de Desarrollo de la Investigación, 1982, $384 \mathrm{pp}$.

Proyectos de Investigación 1983, Universidad de Chile, Dirección General Académica, Departamento de Investigación y Bibliotecas, 1983.

Proceso de Admisión a la Educación Superior, Postulantes y postulaciones año académico 1982, Universidad de Chile, 1982.

Publicaciones Cientificas 1983, Universidad de Chile, Instituto de Nutrición y Tecnología de los Alimentos, Santiago, Chile, 1984.

El perfeccionamiento pedagógico en la Universidad de Chile, Universidad de Chile, 1984. 